RESEARCH PAPER

\title{
Validity of self reports in a cohort of Swedish adolescent smokers and smokeless tobacco (snus) users
}

\author{
A Post, H Gilljam, I Rosendahl, L Meurling, S Bremberg, M R Galanti
}

Tobacco Control 2005;14:114-117. doi: 10.1136/tc.2004.008789

See end of article for authors' affiliations

\section{Correspondence to:} Ann Post, Stockholm Centre of Public Health, Centre for Tobacco

Prevention, Box 17533 S-11891 Stockholm, Sweden; ann.post@smd. sll.se

Received 12 May 2004 Accepted 2 January 2005
Objective: To validate self reports of cigarette and smokeless tobacco (snus) use in a prospective cohort of adolescents.

Design: A cross sectional analysis of a cohort sub-sample.

Setting: County of Stockholm, Sweden.

Subjects: 520 adolescents in the final grade of junior high school (mean age 15.0 years).

Main outcome measure: Concordance between self reported tobacco use and saliva cotinine concentration.

Results: Using a cut point of $5 \mathrm{ng} / \mathrm{ml}$ saliva cotinine to discriminate active tobacco use, there was a $98 \%$ concordance between self reported non-use in the past month and cotinine concentration. The sensitivity of the questionnaire compared to the saliva cotinine test, used as the gold standard, was $90 \%$ and the specificity $93 \%$. One hundred and fifteen out of 520 subjects (22\%) reported monthly tobacco use. Among these, $67 \%(46 / 69)$ of the exclusive cigarette smokers, $82 \%(23 / 28)$ of exclusive snus users, and $94 \%$ $(15 / 16)$ of mixed users (cigarettes + snus) had cotinine concentrations above $5 \mathrm{ng} / \mathrm{ml}$. Among subjects reporting daily use $96 \%$ (64/67) had saliva cotinine concentrations above the cut point. Exclusive current cigarette users were more likely to be classified discordantly by questionnaire and cotinine test compared to snus users (odds ratio $3.2,95 \%$ confidence interval 1.2 to 8.6 ).

Conclusion: This study confirms the reliability of adolescents' self reported tobacco use. In a context of low exposure to environmental tobacco smoke a cut off for saliva cotinine of $5 \mathrm{ng} / \mathrm{ml}$ reliably discriminated tobacco users from non-users. Irregular use of tobacco in this age group probably explains the discrepancy between self reported use and cotinine concentrations.
$\mathrm{T}$ he validity of self reports of smoking among adolescents has been questioned on the grounds that socially unacceptable behaviours would likely be underreported. ${ }^{1}$ Biochemical verification with a highly sensitive method is therefore recommended to ensure that self reports are accurate. Cotinine, a major metabolite of nicotine, has been considered to be the "gold standard" for measuring nicotine intake. ${ }^{2}$ The analysis of saliva cotinine is a routine procedure in adolescent smoking studies and has been shown to provide reliable information on plasma cotinine whether measured in saliva or blood. ${ }^{3}$ Adolescents' self reports have been found to correspond very well with several biochemical markers of tobacco smoking in observational studies when confidentiality was ensured. ${ }^{4}$ However, most studies where the reliability of adolescents' self reports of smoking was assessed were based on populations in which the smoking prevalence was high and the social norms surrounding tobacco less negative than they are today. In countries like Sweden, where a comprehensive Tobacco Act has been implemented and the prevalence of cigarette smoking in the adult population is declining, it might be particularly sensitive for teenagers to report smoking cigarettes. ${ }^{5}$ Moreover, the reliability of self reports of smokeless tobacco use, a very frequent behaviour among Swedish male adolescents, has not been extensively studied. ${ }^{6}$ This study was conducted to assess the reliability of reports of smoking and snus (the Swedish variety of oral moist snuff) use within the frame of a large cohort of Swedish adolescents.

\section{METHODS}

The ethical board of Karolinska University Hospital approved the study.

\section{Subjects}

BROMS (Swedish acronym for Children's Smoking and Environment in the Stockholm County) is a prospective cohort study in the region of Stockholm, encompassing about 3000 children recruited in the spring of 1998 with follow ups 1999 to $2004 .^{7}$ The study was initiated to assess determinants of uptake of tobacco use. The data were collected from annual surveys, using a self completed questionnaire covering questions on health behaviour and tobacco use. For the present study, a sub-sample of the original cohort was selected, consisting of 583 cohort members from a random sample of 18 schools with at least five members in the BROMS study. At the time of the study (2002) the students were in the final grade of junior high school, with a mean age of 15 years. Sixteen pupils did not take the test (three refusals, 12 absentees, and one not located), four subjects did not provide enough saliva to be analysed, and 43 did not complete the questionnaire form. The final sample included in the analysis consisted of 520 subjects with valid questionnaire data and saliva cotinine value-that is, $89 \%$ of the original sample.

\section{Tobacco use and exposure to ETS}

Lifetime use of tobacco was investigated by the following questions: "Did you ever try smoking a cigarette, even a puff/ ever try snus?" (Yes/No). "Did you smoke a whole cigarette in your life?"(Yes/No). Current tobacco use was assessed by the questions "Do you smoke/use snus at present?" with alternatives "Not at all", "Less than once a month", "Every month, but less than one cigarette per week", "Each week, but less than one cigarette per day" and "At least one cigarette every day". A reported frequency of tobacco use of 
at least once a month was considered as current use. The following question was used to estimate recentness of use: "When did you last smoke/use snus?" with seven response alternatives ranging from "today" to "more than one month ago". Recent use (that is, use in the window of detectable cotinine concentration) was categorised as any use in the week before the survey. Regular users (that is, subjects reporting at least weekly use) were asked "How many cigarettes do you usually smoke/how frequently do you use snus during one week?" to assess intensity of use.

In order to rule out exposure to environmental tobacco smoke the following question was asked: "During the last week, approximately how many hours have you spent indoors in a place where someone smoked or had recently smoked?" The alternatives for estimated time of exposure were $0,1-2,3-4,5-7,8-14$, and $>14$ hours for each of the following environments: (1) In your home; (2) In someone else's home; (3) During school hours; (4) In a café, restaurant, pub, discotheque etc; (5) In a car; (6) Elsewhere.

\section{Collection of saliva specimens}

Five months before the survey the selected adolescents and their parents received a letter explaining the purpose, but not the time, of the test. The free choice and the confidentiality of the results were underlined. Consent was assumed in all cases of no explicit refusal. The questionnaire survey was conducted at school, using the same procedure as in previous years. At the time of the survey, the students were not reminded of the coming test. Nevertheless, the samples were taken either later the same day or, with few exceptions, in the next few days. The median time between questionnaire and saliva sampling was 2.0 days (range 0-21 days).

Saliva was chosen as the medium because the non-invasive procedure is more acceptable in this age group. A team consisting of dental hygienists assisted by the school nurse collected the saliva specimens. The pupils were asked to rinse the mouth with water (in order to avoid possible contamination with snus) and to chew on a roll of cotton, which was then spat directly into a test tube, marked with an individual study code. The tubes were brought to the laboratory the same day, centrifuged, and stored at $-80^{\circ} \mathrm{C}$ until analysis. The school nurses took saliva samples on later occasions from students who were absent from school during the test day. No feedback was given to the study subjects or to the schools concerning the survey or the test results.

\section{Saliva cotinine assay}

To identify tobacco users among adults a cut point of $15 \mathrm{ng}$ cotinine $/ \mathrm{ml}$ saliva is customary. ${ }^{8}$ Owing to the low intensity of smoking in this age group, and the low exposure to environmental smoke in Swedish homes, a cut off at $5 \mathrm{ng} / \mathrm{ml}$ was chosen as a threshold for active tobacco use (N Benowitz, personal communication with MR Galanti). ${ }^{9}$ Values of less than $5 \mathrm{ng} / \mathrm{ml}$ were thus interpreted as no tobacco use in the preceding seven days, or low level exposure to passive smoking only. Cotinine in saliva has a half life of 16-20 hours with approximately 100 hours from intake to the chosen cut off value, and was determined by gas chromatography-mass spectrometry (GC-MS), using the method of Curvall $e t a l^{3}$ with cotinine- $\mathrm{d}_{3}$ as an internal standard. LOD (limit of detection) was $0.2 \mathrm{ng} / \mathrm{ml}$ and LOQ (limit of quantitation) $2 \mathrm{ng} / \mathrm{ml}$. Precision of the method was determined by running controls at three levels during three consecutive days. Calibration curves were constructed each day. Within-day precision was determined to coefficient of variation $(\mathrm{CV})$ of: $4.7 \%$ at $5 \mathrm{ng} / \mathrm{ml}(\mathrm{n}=5) ; 4.8 \%$ at $100 \mathrm{ng} / \mathrm{ml}(\mathrm{n}=5) ;$ and $2.9 \%$ at $300 \mathrm{ng} / \mathrm{ml} \quad(\mathrm{n}=5)$. Between-day precision was determined to $4.53 \%$ at $5 \mathrm{ng} / \mathrm{ml}$ $(\mathrm{n}=15) ; 5.82 \%$ at $100 \mathrm{ng} / \mathrm{ml}(\mathrm{n}=15) ;$ and $4.1 \%$ at

\begin{tabular}{|c|c|c|}
\hline & Number & $\%$ \\
\hline \multicolumn{3}{|l|}{ Sex } \\
\hline Boys & 255 & 49.0 \\
\hline Girls & 265 & 51.0 \\
\hline \multicolumn{3}{|c|}{ Parental education index* } \\
\hline Compulsory school & 61 & 11.8 \\
\hline College & 202 & 39.2 \\
\hline University & 252 & 48.5 \\
\hline \multicolumn{3}{|l|}{ Lifetime use of tobacco } \\
\hline None & 134 & 25.8 \\
\hline Snus only & 54 & 10.4 \\
\hline Smoking only & 114 & 21.9 \\
\hline Snus and smoking & 218 & 41.9 \\
\hline
\end{tabular}

$300 \mathrm{ng} / \mathrm{ml}(\mathrm{n}=15)$. Calibration curves had always correlation constants better than 0.998 and varied marginally in slope between days.

\section{Statistical methods}

For the purpose of assessing reliability of self reports, we primarily conducted a descriptive analysis by comparing the expected proportion of cotinine-negative among self reported non-users (expected 100\%) and of cotinine-positive among self reported current users.

We also analysed the same proportions separately by type of tobacco used. In tabular data, the departure of the observed from the expected distribution was formally tested by means of $\chi^{2}$ statistics. We also analysed three categorical predictors of discordance between self reported tobacco use and classification on the cotinine test, namely type of product (cigarettes only or snus, alone or in combination with cigarettes), intensity of use (no weekly use, 1-30 or more than 30 cigarettes/snus dips per week); and recentness of use (within one day, 2-7 days, or $>7$ days before the survey). As measures of association odds ratios (OR) and their corresponding 95\% confidence intervals (CI) were computed by ordinary logistic regression. This analysis was conducted both among all subjects and after restriction to current users. The level for the significance was conventionally set at 5\% $(\mathrm{p}<0.05)$.

All data analyses were conducted using the SPSS 10.0.5 for Windows (SPSS Inc, Chicago, Illinois, USA).

\section{RESULTS}

The characteristics of the study sample are shown in table 1. At the time of the survey, 386 subjects (74.2) had at least tried tobacco, $115(22.1 \%)$ were current users (reported use at least monthly), and 102 (19.7\%) were recent users (use in the week before the survey). The sensitivity and specificity of the questionnaire compared to the saliva cotinine test (gold standard) were $90 \%$ and $93 \%$, respectively, at the cut off of $5 \mathrm{ng} / \mathrm{ml}$. The overall concordance between the two measures was $93 \%$. At the most commonly adopted cut off, $15 \mathrm{ng} / \mathrm{ml}$, the questionnaire had a sensitivity of $93 \%$ and a specificity of $92 \%$. Table 2 shows the cross classification of the cotinine test by self reported use. Ninety-eight per cent of subjects reporting no use or less than monthly use had a saliva cotinine concentration lower than $5 \mathrm{ng} / \mathrm{ml}$. Of the nine subjects with saliva cotinine concentration above $5 \mathrm{ng} / \mathrm{ml}$ (false negative according to the questionnaire), seven reported exposures to environmental smoke and three subjects reported recent use (past week). Among daily users about $96 \%$ had concentrations above cut-off. Among subjects reporting weekly, but not daily, use, every second adolescent had a cotinine concentration below the cut off (false positives). A comparison between the two different sets of 
Table 2 Saliva cotinine concentrations according to self reported current tobacco use

\begin{tabular}{|c|c|c|c|c|c|c|c|c|c|c|}
\hline \multirow{3}{*}{$\begin{array}{l}\text { Saliva cotinine } \\
\text { (ng/ml) }\end{array}$} & \multicolumn{10}{|c|}{ Current self reported tobacco use } \\
\hline & \multicolumn{2}{|c|}{$\begin{array}{l}\text { Not at all or less than } \\
\text { every month }\end{array}$} & \multicolumn{2}{|c|}{ Monthly, less than weekly } & \multicolumn{2}{|c|}{ Weekly, not daily } & \multicolumn{2}{|l|}{ Daily } & \multicolumn{2}{|l|}{ Total } \\
\hline & Number & $\%$ & Number & $\%$ & Number & $\%$ & Number & $\%$ & Number & $\%$ \\
\hline$\leqslant 5$ & 395 & 97.8 & 9 & 69.2 & 18 & 51.4 & 3 & 4.5 & 425 & 81.9 \\
\hline$>5$ & 9 & 2.2 & 4 & 30.8 & 17 & 48.6 & 64 & 95.5 & 94 & 18.1 \\
\hline Total & 404 & 100 & 13 & 100 & 35 & 100 & 67 & 100 & 519 & 100 \\
\hline
\end{tabular}

questions concerning tobacco use revealed that "noncurrent" users also reported being "non-recent" users in $389 / 404$ (96\%) of cases (data not shown).

Among current users $69(61.0 \%)$ were exclusive smokers, $28(24.8 \%)$ used only snus, and $16(14.2 \%)$ used both types of tobacco (two subjects had incomplete data). The tobacco consumption reported by regular smokers (mean 47 cigarettes/week), snus users (mean 31 pinches/week), and mixed users (mean 66 cigarettes or pinches/week) correlated significantly to saliva cotinine concentration (Spearman $r=0.42 ; \mathrm{p}<0.01$ ) (data not shown).

The distribution of saliva cotinine concentration among current users is shown in fig 1 . The median concentration was significantly different between exclusive smokers, exclusive users of snus, and mixed users. Concordantly, there was a significant difference in the proportions classified as positive by the cotinine test among self reported users (table 3). In fact, one third of the smokers had cotinine concentrations below the threshold, while this was found in nearly one out of six snus users and for only one in 16 mixed users.

In analyses using logistic regression, current users had a higher likelihood of discordance between self reports and cotinine tests, compared to non-users. Predictors of discordance were also: recentness (use not occurring the very same day of the survey); use of a moderate amount of tobacco per week (1-30 pieces-that is, cigarettes and snus dips) compared to heavier use (more than 30 tobacco pieces per week), and patterns of product use. Exclusive cigarette users were more likely to be classified discordantly by the questionnaire versus cotinine test compared to snus and mixed users (OR 3.2, 95\% CI 1.2 to 8.6), even after

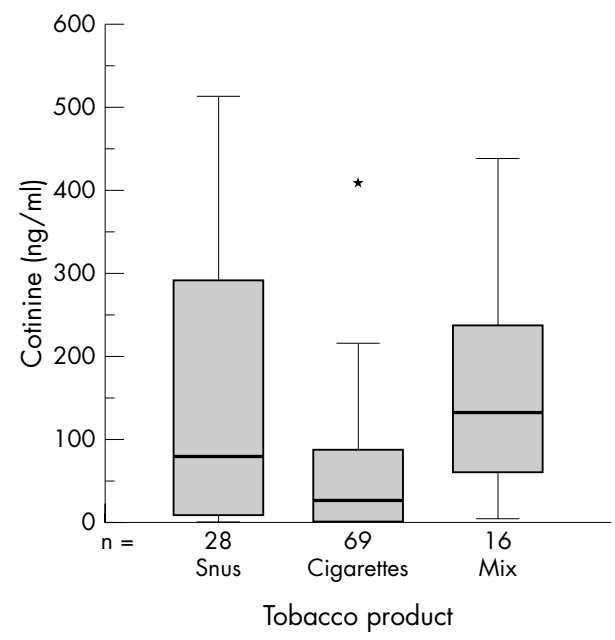

Figure 1 Quartiles, mean, and range of cotinine concentrations among current (at least monthly) users of the different tobacco products. Kruskall-Wallis test for difference between medians $p<0.001$. *Extreme value.
Table 3 Saliva cotinine concentrations according to monthly users of cigarettes and snus

\begin{tabular}{|c|c|c|c|c|c|c|}
\hline \multirow{2}{*}{$\begin{array}{l}\text { Saliva } \\
\text { cotinine } \\
\text { (ng/ml) }\end{array}$} & \multicolumn{2}{|c|}{ Only cigarettes } & \multicolumn{2}{|c|}{ Only snus } & \multicolumn{2}{|c|}{$\begin{array}{l}\text { Both cigareftes } \\
\text { and snus }\end{array}$} \\
\hline & Number & $\%$ & Number & $\%$ & Number & $\%$ \\
\hline$\leqslant 5$ & 23 & 33.3 & 5 & 17.9 & 1 & 6.2 \\
\hline$>5$ & 46 & 66.7 & 23 & 82.1 & 15 & 93.8 \\
\hline Total & 69 & 100 & 28 & 100 & 16 & 100 \\
\hline
\end{tabular}

adjustment for quantity or recentness of use. The adjusted estimates, however, were imprecise because of the paucity of the observations.

\section{DISCUSSION}

In this sample of Swedish 15 year olds there was a high overall correspondence between self reported tobacco use and use assessed by saliva cotinine. Indeed, the correspondence was higher for self reported non-users than for users. Overall, the correlation between the biological marker and the reported patterns of use (frequency, recentness, and intensity) was very good. A considerable discrepancy was found among subjects reporting non-daily tobacco use: half of the weekly users, for example, were classified as non-users by the biological test. The discrepancy is likely explained by the very unstable patterns of tobacco use in this age group, for which recent consumption does not imply intensive, nor regular use. ${ }^{10}$ In fact, infrequent and low level users in this cohort were less likely to be consistently classified by the two instruments than non-users or heavy/frequent users. It should also be noted that patterns of inhalation might vary substantially in this age group, not only between subjects, but also within subjects on different occasions. ${ }^{11}$ Our study also shows that recent and habitual use probably capture the same conceptual definition of intensity of use-in fact, virtually all of "non-current" users were also "non-recent" users. More self reported smokers than snus users or combined users fell below the cut off value of the biological marker, and were therefore classified as non-users. To our knowledge this comparison has not been reported before. Since there is no reason to believe that smokers, more than snus users, would intentionally exaggerate their tobacco consumption, this difference may be explained in terms of frequency/intensity of use. However, in this study the type of tobacco used remained a predictor of discordance between self report and biological classification, even after adjustment for quantity or recentness of use, indicating that factors connected to topography of use and/or uptake of nicotine also play a role. For example, the exposure to nicotine from snus (a pinch of tobacco put under the lip and left in place for approximately 30 minutes) is likely to be more prolonged than that occurring after cigarette smoking. Although not a primary interest in this study, this finding deserves further 


\section{What this paper adds}

In general, the validity of adolescent self reported smoking has been found to be accurate whereas little is known about smokeless tobacco users. Discordant results between the biological marker and reports could be explained by, for example, fear of breaking confidentiality, unstable patterns of tobacco use, lack of specificity of questionnaires, or by the biological indicator examined.

This is the first biochemically verified study of self reports among Swedish adolescent smokers and smokeless tobacco (snus) users. Using a cut point of only $5 \mathrm{ng} / \mathrm{ml}$ saliva cotinine to identify active tobacco use, there was a $98 \%$ concordance between self reported non-use and cotinine concentration. More self reported smokers than snus users or combined users fell below the cut off value of the biological marker. The type of tobacco used remained a predictor of discordance between self report and biological classification, even after adjustment for quantity or recentness of use.

attention because of its obvious implication for the natural history of nicotine dependence among young snus users.

This study has some weaknesses. First, it was not possible to standardise the time interval between self report and cotinine test, since the test procedure had to be coordinated with the annual survey. This may have caused some occasional users to be misclassified as non-users by the biological marker. At the time of the survey the study subjects were almost certainly not aware that the test was soon going to be performed, because the information received some months earlier was not repeated, and was never announced at the time of the test. This makes the possibility of behavioural or report modification unlikely. This possibility cannot be ruled out for school absentees tested at a later date, but very few subjects fell into this group. Because of the relatively low prevalence of regular tobacco use among the Swedish youths there were few frequent users, a fact that hampered a more refined analysis of the reliability of the reported intensity of use.

Strengths of the study included the very high participation rate, the detailed history of both cigarette smoking and smokeless tobacco use, as well as the very sensitive assay employed for the test. The set of questions tackling tobacco use in the annual questionnaire seems to classify correctly the different tobacco patterns typical of adolescence. This is, to our knowledge, the first study of biochemical verified self reports of tobacco use among Swedish adolescents and one of few internationally published covering smokeless tobacco use. $^{12}$

Above all this study shows that adolescents give valid reports of tobacco use. Our results also suggest that the high reliability already observed in other youth studies is not affected by strongly negative societal attitudes towards tobacco use, cigarette smoking in particular. ${ }^{13} \mathrm{~A}$ cut off of $5 \mathrm{ng} / \mathrm{ml}$ discriminated tobacco users from non-users in this study without appreciable loss in sensitivity and specificity compared to the most commonly adopted cut off of $15 \mathrm{ng} / \mathrm{ml}$.
The generally low exposure to passive smoking in Sweden, especially when children and adolescents are concerned, might also have contributed to the very good correspondence between gold standard and self reports at a very low cut off. ${ }^{14}$ The question of cost effectiveness of biological validation of tobacco use in future adolescents' studies should therefore be considered.

\section{ACKNOWLEDGEMENTS}

The authors wish to express sincere appreciation to the students, teachers, and nurses in the cooperating schools. Thanks to Åsa Forsgård, Inga-Lena Sjögren, and Renée Stockling for their work with the specimens collection, and to Mats Toftgård for helping in the analysis of the data.

\section{Authors' affiliations}

A Post*, H Gilljam*, S Bremberg, Department of Public Health Sciences, Division of Social Medicine, Karolinska Institute, Stockholm, Sweden

\section{Rosendahl}

†, M R Galantił , Centre for Tobacco Prevention, Stockholm County Council, Stockholm, Sweden

L Meurling, Department of Clinical Pharmacology, Karolinska University Hospital, Stockholm, Sweden

* Also Centre for Tobacco Prevention, Stockholm County Council † Also Division of Epidemiology, Institute of Environmental Medicine, Karolinska Institute

$\ddagger$ Also Clinical Epidemiology Unit, Department of Medicine, Karolinska Institute

\section{REFERENCES}

1 Evans RI, Hansen WB, Mittelmark MB. Increasing the validity of self-reports of smoking behavior in children. J Appl Psychol 1977;62:521-3.

2 Benowitz NL, Kuyt F, Jacob P, et al. Cotinine disposition and effects. Clin Pharmacol Ther 1983;34:604-11.

3 Curvall M, Elwin CE, Kazemi-Vala E, et al. The pharmacokinetics of cotinine in plasma and saliva from non-smoking healthy volunteers. Eur J Clin Pharmacol 1990;38:281-7.

4 Mayhew KP, Flay BR, Mott JA. Stages in the development of adolescent smoking. Drug Alcohol Depend 2000;59(suppl 1):S61-81.

5 Statistics Sweden. Tobakskonsumtionen 1980-2002 (Tobacco consumption 1980-2002). Statistics Sweden, 2002.

6 Galanti MR, Wickholm S, Gilljam H. Between harm and dangers. Oral snuff use, cigarette smoking and problem behaviours in a survey of Swedish male adolescents. Eur J Public Health 2001;11:340-5.

7 Galanti MR, Rosendahl I, Post A, et al. Early gender differences in adolescent tobacco use--the experience of a Swedish cohort. Scand J Public Health 2001;29:314-7.

8 SRNT Subcommittee on Biochemical Verification. Biochemical verification of tobacco use and cessation. Nicotine Tob Res 2002;4:149-59.

9 Helgason AR, Lund KE. Environmental tobacco smoke exposure of young children--attitudes and health-risk awareness in the Nordic countries. Nicotine Tob Res $2001 ; 3: 341-5$

10 Williams CL, Eng A, Botvin GJ, et al. Validation of students' self-reported cigarette smoking status with plasma cotinine levels. Am J Public Health 1979;69:1272-4

11 Dolcini MM, Adler NE, Lee $P$, et al. An assessment of the validity of adolescent self-reported smoking using three biological indicators. Nicotine Tob Res 2003;5:473-83.

12 Bauman KE, Koch GG, Bryan ES, et al. On the measurement of tobacco use by adolescents. Validity of self-reports of smokeless tobacco use and validity of cotinine as an indicator of cigarette smoking. Am J Epidemiol 1989;130:327-37.

13 Caraballo RS, Giovino GA, Pechacek TF. Self-reported cigarette smoking vs. serum cotinine among U.S. adolescents. Nicotine Tob Res 2004;6:19-25.

14 Willers S, Axmon A, Feyerabend C, et al. Assessment of environmental tobacco smoke exposure in children with asthmatic symptoms by questionnaire and cotinine concentrations in plasma, saliva, and urine. J Clin Epidemiol 2000;53:715-21. 